\title{
Recurrent pulmonary thromboembolism presenting with cardiac arrhythmias
}

\author{
A K BROWN, P NEWTON, E A G HAMILTON, AND V ANDERSON \\ From the Royal Lancaster Infirmary, Lancaster, UK
}

ABSTRACT Pulmonary emboli seldom recur, and when recurrence does occur it is not associated with permanent sequelae unless there is progressive pulmonary arterial hypertension. Five patients with clinical and perfusion lung scan evidence of recurrent pulmonary embolism presented with abnormal cardiac rhythms without evidence of progressive pulmonary hypertension. Twenty-four-hour ambulatory electrocardiographic monitoring was valuable in diagnosis and in assessing the effectiveness of treatment. Although palpitation was the main complaint, other symptoms included tiredness, mild exertional dyspnoea, and chest discomfort unrelated to effort. Symptomatic improvement coincided with objective evidence of improvement from repeat lung scans and 24-hour ECG records. Antiarrhythmic agents controlled the arrhythmias but were subsequently withdrawn without the return of symptoms. Four of the five patients continued to take anticoagulants for two years. We believe that these five patients represent a group of patients with recurrent pulmonary emboli and a recognisable clinical picture dominated by arrhythmias unrelated to progressive pulmonary arterial hypertension. Long-term anticoagulant treatment was associated with clinical improvement.

There is little information about the long-term sequelae in patients who survive pulmonary embolism (Lancet, 1978). Although Genton (1973) has suggested that most emboli that are recognised represent recurrence rather than the first episode, most authors agree that recurrent pulmonary embolism is rare, and resolution occurs within four months with subsequent normal haemodynamic and angiographic findings in most treated patients (Lancet, 1978). Progression to pulmonary arterial hypertension has been recorded (Goodwin et al, 1963; Fleischner, 1967) but repeated emboli usually resolve without serious symptoms or pulmonary hypertension (Oakley, 1977). We describe five patients with evidence of recurrent pulmonary thromboembolism who presented with abnormal cardiac rhythms.

\section{Case reports}

\section{CASE 1}

A woman aged 47 was first seen in June 1974 with a six-month history of bouts of tachycardia and dyspnoea. There were no abnormal signs in the heart or chest, and investigations showed an ESR of $17 \mathrm{~mm} / \mathrm{hr}$ (Westergren). T4 (Thyopac), chest radiograph, and ECG were normal. Pulmonary function tests showed $\mathrm{FEV}_{1} 2.041$ (predicted 2.70) FVC 3.22 1 (predicted 3.40), and transfer factor $4.8 \mathrm{mmol} \mathrm{min}^{-1} \mathrm{kPa}^{-1}$ (predicted 8.3). Twenty-four $-x$ hour ambulatory ECG monitoring using the Oxford Instruments Medilog system showed boutsin of supraventricular tachycardia at $190 / \mathrm{min}$ associated with the subjective complaint of palpitations 0 In February 1975 pulmonary arteriography showedP relative avascularity of the left lower lobe, anto the lung scan showed poor perfusion of the left lower lobe and multiple perfusion defects in thes right lung compatible with pulmonary emboli.

The major occlusion of the artery to the lef? lower lobe was thought to have occurred aftef hysterectomy in 1966 for carcinoma of the cervix? A postoperative diagnosis of left lower lobe colo lapse had been reported in the notes. There was no evidence of recurrent carcinoma at annual examinations to 1978 .

Treatment with warfarin and propranolol did not reduce the bouts of tachycardia, and subse $\frac{\rho}{\Phi}$ quently verapamil and amiodarone were also trieç without success. On two occasions, prolonge $\bar{R}$ 
bouts of atrial fibrillation were recorded and digoxin was prescribed for three months before the patient stopped the drug. Spontaneous improvement in the dyspnoea and tachycardia occurred and, when seen in March 1976, there was mild dyspnoea on exertion only, and a repeat lung scan showed only the left lower lobe perfusion defect. Warfarin was discontinued in December 1977, and the arrhythmias have not recurred.

\section{CASE 2}

In 1973 a 49-year-old woman presented with ankle and leg oedema and obesity. She was considered to have lymphoedema praecox, and results of routine clinical examination, chest radiograph, and ECG were normal.

In October 1973 she was seen again with a deep vein thrombosis of the right leg and trans-sternal chest pain. Blood pressure was $125 / 70 \mathrm{mmHg}$ and chest radiograph was normal. A standard 12 lead ECG showed $T$ wave inversion in leads III and AVF, and she was treated with warfarin for six months. In June 1974 she started having bouts of palpitations lasting up to half an hour, and mild chest discomfort was reported with the more prolonged bouts. Digoxin partially controlled the attacks, but in August 1975 she was admitted to hospital with atrial fibrillation. A perfusion lung scan was normal, as was pulmonary angiography and peripheral venography in February 1976. Three months later she was readmitted to hospital with chest pain and haemoptysis, and the chest radiograph was thought to be compatible with a pulmonary embolus. Warfarin was restarted but she continued to complain of palpitations. In May 1977 24-hour ambulatory ECG monitoring showed bouts of supraventricular tachycardia and atrial fibrillation. Metoprolol was started and a repeat 24-hour ECG showed only ventricular and juntional premature beats. Spirometry showed an $\mathrm{FEV}_{1}$ of 3.1 1 (predicted 3.4) and FVC of 3.61 (predicted 3.9).

Tiredness and moderate dyspnoea on exertion were the complaints at review in May 1978 and no tachycardia had been reported in the previous four months. Metoprolol was withdrawn but warfarin was continued, and repeat chest film and perfusion lung scan were normal.

\section{CASE 3}

In October 1976 a woman of 56 presented with a six-month history of bouts of palpitations and chest discomfort associated with moderate dyspnoea on exertion. She gave a past history of varicose vein ligation 22 years previously after recurrent thrombophlebitis after pregnancy three years before. There was a possible story of deep vein thrombosis in 1974 associated with pleuritic pain. Examination showed no abnormalities in the heart or lungs, and the blood pressure was $130 / 70 \mathrm{mmHg}$. Chest radiograph and resting and postexercise electrocardiograms were normal as was routine spirometry.

Perfusion lung scanning in November 1976 showed multiple defects compatible with pulmonary emboli, and she began taking propranolol and warfarin.

In May 1977 she had fewer bouts of tachycardia but complained of sudden attacks of fatigue. A 24-hour ambulatory ECG showed multifocal premature ventricular beats and Möbitz type II atrioventricular block coinciding with the periods of weakness, and numerous unifocal ventricular premature beats at other times on the record. Mexilitine was substituted for propranolol and the symptoms settled. A repeat lung scan in April 1978 was normal and mexilitine and warfarin have been continued.

\section{CASE 4}

In July 1975 a woman aged 43 was seen with tiredness, mild exertional dyspnoea, and illdefined palpitations. Left chest pain unrelated to exertion or respiration was admitted on direct questioning. The only abnormal finding on routine examination was bilateral varicose veins. She gave a history of pleurisy 26 years previously. A resting ECG, chest radiograph, and spirometry were normal. Perfusion lung scan in September 1975 showed multiple defects, and warfarin was started.

After six months warfarin was discontinued, and three months later she presented with a painful swollen right leg and pleuritic left chest pain. The chest film was compatible with a left lower lobe pulmonary infarct, and warfarin was restarted. Subsequently, repeated, short lasting bouts of palpitations and exhaustion restricted normal life and 24-hour ambulatory ECG monitoring in May 1977 showed frequent supraventricular tachycardia at $180-200 / \mathrm{min}$. Treatment with atenolol, $100 \mathrm{mg}$ daily, rapidly improved the symptoms and the beta-blocker was withdrawn in February 1978 without clinical deterioration. Repeat perfusion lung scanning in April 1978 was normal, and warfarin was continued.

\section{CASE 5}

A 48-year-old woman was first seen in July 1976 with a 12-month history of tiredness and slight dyspnoea on effort, and for six months she had had chest discomfort unrelated to exertion. Chest 
radiograph, ECG, and routine examination showed no abnormalities and the blood pressure was $125 / 75 \mathrm{mmHg}$.

She was seen again in May 1977 with persistence of the previous symptoms plus bouts of palpitations lasting up to half an hour at a time. Clinical examination, chest film, and resting ECG were again normal, but 24-hour ambulatory ECG monitoring showed one run of supraventricular tachycardia coinciding with the subjective sensation of palpitations. Perfusion lung scanning showed multiple perfusion defects compatible with pulmonary thromboemboli. Spirometry showed FEV 2.7 1 (predicted 3.0) and FVC 3.05 1 (predicted 3.6). Warfarin was started, and a repeat lung scan in April 1978 was normal.

\section{Discussion}

Abnormalities of cardiac rhythm are not mentioned in long-term studies of patients with recurrent pulmonary thromboembolism that have concentrated either on patients with chronic pulmonary arterial hypertension or on asymptomatic subjects with repeated peripheral pulmonary emboli with complete resolution (Oakley, 1977). Two clinical forms of chronic thromboembolic pulmonary hypertension have been described (Goodwin et al, 1963; Wilhelmsen et al, 1972). One shows distinct episodes of clinical deterioration often associated with definite pulmonary embolic incidents; the other presents with steady progression of dyspnoea without clear pulmonary embolic episodes. The former is thought to be caused by thromboembolic occlusion of major pulmonary arteries, while the latter is associated with emboli in smaller vessels. Diagnosis of these patients has followed presentation with chronic pulmonary arterial hypertension and prognosis is poor, although a few patients with recurrent major pulmonary arterial thromboembolic disease have responded to anticoagulants.

Recurrence of pulmonary emboli is uncommon, and complete clinical recovery is usual except in the rare cases that progress to pulmonary arterial hypertension. Thus in a series of 46 patients without cardiopulmonary disease followed for one to nine years after massive pulmonary embolism, only one patient has suffered recurrent embolism (Hall et al, 1977). The same workers reported three of 13 patients with acute minor pulmonary emboli with recurrent pulmonary embolism one, two, and five years after the first episode (Sutton et al, 1977). Although perfusion lung scans often remained abnormal, no patient in these two series had progressive pulmonary arterial hypertension.
Similarly, Paraskos et al (1973) reported $43 \stackrel{:}{\Rightarrow}$ patients who had been followed up for one to $\stackrel{\mathcal{P}}{+}$ seven years after pulmonary emboli. One patient $\frac{}{c}$ developed chronic cor pulmonale, and five had evidence of unresolved embolism. Recurrent pul- $\frac{\infty}{\square}$ monary emboli were suspected in four patients.

There was no clinical, electrocardiographic, or \& radiographic evidence of pulmonary arterial $\vec{\circ}$ hypertension in our patients, either on presentation or during the period of study which has been $\vec{\omega}$ from two to five years. Although all were women, $\stackrel{\circ}{\circ}$ none had taken the contraceptive pill. Confirma- $\vec{x}$ tion of multiple thromboembolic incidents depends $\omega$ on perfusion lung scanning, and since abnormal $\stackrel{\oplus}{\omega}$ perfusion scans may be caused by ventilatory ab- $\omega$ normalities as well as pulmonary arterial obstruc- ${ }^{\circ}$ tion (Critchley and Bone, 1978), respiratory을 function studies were performed with normal results in each case. The abnormal perfusion lung $\subseteq$ scans showed multiple discrete perfusion defects, $\underset{\nabla}{\overparen{D}}$ but we have been unable to separate patients with $\vec{\varphi}$ large defects from those with small defects (Wilson et al, 1973), and variable sizes of defects are present in each case.

Concomitent coronary artery disease could be postulated as a cause of the arrhythmias, but no patients have had ischaemic pain or ECG changes $\bigcirc$ of ischaemic heart disease at rest or on exercise. $\unrhd$ Moreover, clinical improvement has clearly coin- $\overrightarrow{\overrightarrow{0}}$ cided with improvement in the lung scan.

Twenty-four-hour ambulatory ECG monitoring has shown the nature of the abnormal rhythms in all five patients. Special care has been taken to show that complaints of palpitations have coin- 윽 cided with the electrocardiograph arrhythmias $\tilde{x}$ since serious ventricular and supraventricular $\frac{3}{3}$ tachyarrhythmia was recorded by 24-hour-ECG monitoring in 14 of 86 asymptomatic volunteers $O$ by Clarke et al (1976).

Although the patients in this series received 을 antiarrhythmic agents initially, arrhythmia did not $D$ recur when the drugs were withdrawn. The symptomatic improvement coincided with objective $\tilde{\sigma}^{\circ}$ evidence of resolution of defects on repeat perfusion lung scans, and hence we conclude that $\tilde{D}$ there is a strong likelihood that the arrhythmias $\omega$ were started by multiple pulmonary thromboembolic episodes, and that prevention of furthero pulmonary incidents by anticoagulant treatment $\mathbb{\Phi}$ was responsible for the clinical improvement. We $\stackrel{?}{+}$ have no explanation for the production of cardiac $\underline{T}$ arrhythmias by repeated pulmonary emboli, but abnormal rhythms are well recognised in association with acute pulmonary embolism.

We believe that these five patients represent a group of patients with a recognisable clinical pic- 
ture caused by repeated pulmonary thromboemboli which may not progress to obliterative pulmonary arterial hypertension. The presenting clinical feature is abnormal cardiac rhythm, and 24-hour-ambulatory ECG monitoring may be required for diagnosis of the arrhythmia. Associated non-specific symptoms may include tiredness, mild exertional dyspnoea, and chest discomfort unrelated to exertion, and the history may show previous peripheral venous problems or possible embolic incidents. Prolonged treatment with anticoagulants is necessary, and our policy has been to continue warfarin for a minimum of two years.

\section{References}

Clarke, J M, Hamer, J, Shelton, J R, Taylor, S, and Venning, G R (1976). The rhythm of the normal human heart, Lancet, 2, 508-512.

Critchley, M, and Bone, D (1978). Differentiation of pulmonary embolism from chronic airways obstruction by a dual isotope technique. Journal of the Royal College of Physicians of London, 12, No 2, 161-168.

Fleischner, F G (1967). Recurrent pulmonary embolism and cor pulmonale. New England Journal of Medicine, 276, 1213-1220.

Genton, E (1973). Recognising and reacting to recurrent pulmonary embolism. Geriatrics, 28, 84-88.

Goodwin, J F, Harrison, C V, and Wilcken, D E L
(1963). Obliterative pulmonary hypertension and thromboembolism. British Medical Journal, 1, 701711.

Hall, R J C, Sutton, G C, and Kerr, I H (1977). Longterm prognosis of treated acute massive pulmonary embolism. British Heart Journal, 39, 1128-1134.

Lancet (1978). What happens to clots in the lungs? 1, 194-195.

Oakley, C (1977). The diagnosis of acute pulmonary embolism. British Journal of Hospital Medicine, 18, 15-24.

Paraskos, J A, Adelstein, S J, Smith, R E, Rickman, F D, Grossman, W, Dexter, L, and Daleh, J E (1973). Late prognosis of acute pulmonary embolism. New England Journal of Medicine, 289, 55-62.

Sutton, G C, Hall, R J C, and Kerr, I H (1977). Clinical course and late prognosis of treated subacute massive, acute minor and chronic pulmonary thromboembolism. British Heart Journal, 39, 1135-1142.

Wilhelmsen, L, Hagman, M, and Werkö, L (1972). Recurrent pulmonary embolism-incidence, predisposing factors and prognosis. Acta Medica Scandinavica, 192, 565-575.

Wilson, A G, Harris, C N, Lavender, J P, and Oakley, C M (1973). Perfusion lung scanning in obliterative pulmonary hypertension. British Heart Journal, 35, 917-930.

Requests for reprints to: $\operatorname{Dr}$ Allen $\mathrm{K}$ Brown, Royal Lancaster Infirmary, Lancaster. 